\title{
Factors Challenge Oropharyngeal and Nasopharyngeal Swab Results in COVID-19 patients
}

\author{
Akif İșlek $^{1 *}$ and Mustafa Koray Balci ${ }^{2}$ \\ ${ }^{1}$ Nusaybin State Hospital, Otolaryngology-Head \& Neck Surgery Clinic, Mardin, Turkey \\ ${ }^{2}$ Katip Celebi University, Atatürk Training and Research Hospital, Otolaryngology-Head \& Neck Surgery Clinic, Izmir, Turkey
}

*Corresponding author: Akif İşlek, Nusaybin State Hospital, Otolaryngology-Head \&

Neck Surgery Clinic, Mardin, Turkey.

\section{Abstract}

Introduction: The aim of this study is to investigate the effect of possible local factors on oropharyngeal and nasopharyngeal swabs (OP/NP) in COVID-19 patients.

Materials and methods: The study was designed retrospectively. The demographic characteristics, symptoms, medical history, chronic diseases, and prescriptions, OP/NP sample collectors [otorhinolaryngologist (ORL) or other physicians (OP)], sampling time, reverse-transcription polymerase chain reaction (RT-PCR) test results of the patients were scanned. Presence of laryngopharyngeal reflux (LPR), allergic respiratory diseases (ARD), nasal steroid use (NSU) was identified.

Results: 68 patients included in the study. NSU, LPR, and sample collectors except for otorhinolaryngologist (ORL) were significant for the negative $\mathrm{OP} / \mathrm{NP}$ swap results (respectively $\mathrm{p}=0.035,0.033$, and 0.012 , respectively $\chi 2$-test). All samples were collected on average $3.8 \pm 2.2$ days after the onset of symptoms and sampling time was not a significant factor for RT-PCR results. Gender and ARD were not found as a significant factor for the first RT-PCR results ( $\mathrm{p}=0.543$ and $0.716, \chi 2$-test). But according to logistic regression analysis, only LPR and sample collector was found significant factors ( $\mathrm{p}=0.043$ and 0.018$)$.

Conclusion: In the presence of LPR and NSU, OP/NP swaps collection can be recommended by an ORL, so the accuracy of the samples can be increased.

Keywords: COVID-19; Nasal steroid; Laryngopharyngeal reflux; SARS-CoV-2; Oropharyngeal swab; Nasopharyngeal swab

\section{Introduction}

SARS-CoV-2 is a coronavirus and caused novel COVID-19 pandemic [1]. The exact diagnosis is made by detecting nucleic acids of the virus by reverse-transcription polymerase chain reaction (RT-PCR) in oropharyngeal and nasopharyngeal (OP/NP) swabs but the sensitivity of the nucleic acid test is closely related to the detection sample [2, 3].However, it is possible to be affected by RT-PCR test due to reasons such as collection time of swabs, correct technique and patient characteristics [3]. Therefore, chest computed tomography (CT) is still more sensitive than a nucleic acid test in the diagnosis of COVID-19 [2]. Laryngopharyngeal reflux (LPR) is a chronic disease that can cause chronic pathological changes in the pharynx and nasopharynx [4-6]. Similarly, allergic rhinitis is a common condition in otorhinolaryngology practice, also patients with allergic rhinitis associated with chronic nasal steroid use (NSU) [7,8]. Recently, it has been confirmed that the immune response and microbial flora of allergic mucosa differ from that of healthy subjects in allergic respiratory diseases (ARD) [9]. It has been proven that budesonide inhibits rhinovirus replication and stimulates cytokine production in primary cultures of human tracheal epithelial cells [10]. 


\section{Materials and Methods}

The study was designed retrospectively. Between 15 March 2020 and 10 April 2020, Patients with clinical, laboratory and radiological accordance for COVID-19 were included in the study at the second referral state hospital. The demographic characteristics of the patients were recorded. Head and neck system-specific and other symptoms were recorded. Afterward, medical history, chronic disease, and prescriptions of patients were scanned retrospectively for six last months. Patients diagnosed with ARD, AR, or LPR and, patients who were orderly prescribed nasal steroids were identified. RT-PCR results were determined as positive or negative by the first sample. All OP/NP samples were collected with a swab and placed in the transport container. After the first negative RTPCR test, NP/OP samples were collected two days later from patients again. Patients who were negative for the second RT-PCR test repeated after 2 days and patients with SARS-CoV- 2 negative in all three tests were excluded from this study.

In addition, sample collectors for OP/NP swaps were grouped as an otorhinolaryngologist (ORL) or other physicians (OP). The frequency of symptoms and categorical variables was calculated in patients. The analysis of categorical dependent variables for RTPCR test results was performed first with the chi-square test $(\chi 2)$, and then logistic regression analysis was performed for significant variables. SPSS 22.0 program (IBM Corp., Armonk, NY, USA) was used for statistics. Verbal consent was obtained from all patients for the study. The study was carried out in accordance with the 1964 Helsinki Declaration and amendments. The institutional review board was approved by the local Provincial Health Directorate for the study (16/04/2020-806.02.02).

\section{Results}

68 patients included in the study. $30(44.1 \%)$ of the patients were female and 38 (55.9\%) were male. The mean age was $51.8 \pm 21.5$ in all patients. The most common symptoms were cough $(n=48,70.6 \%)$, fever $(n=39,57.4 \%)$ and dyspnea $(n=39,57.4 \%)$. The most common symptoms in the head and neck system were sore throat ( $n=25,36.8 \%)$ and smell loss $(n=24,35.3 \%)$ (Table 1$)$.

Samples were collected at average 3.4 \pm 2.1 days from the onset of symptoms from patients with positive RT-PCR tests and on average $4.4 \pm 3.5$ days from patients who were negative. $(p=0.124$, t-test). ARD detected in 15 (22.1\%) patients and LPR was found in $15(22.1 \%)$ patients. The NSU was determined in $12(17.6 \%)$ in patients during the last six mounts. OP/NP swabs were collected by an ORL with a ratio of $30.9 \%(n=21)$ and by OP with a ratio of $69.1 \%(n=47)$. At the first RT-PCR test after hospitalization, 38 (55.9\%) were detected as positive (Table 2). Gender and ARD were not found as a significant factor for the first RT-PCR results ( $p=0.543$ and $0.716, \chi 2$-test). NSU, LPR, and sample collectors were significant for the RT-PCR results (respectively $p=0.035,0.033$ and $0.012, \chi 2$-test) (Table 3). But according to logistic regression analysis, only LPR and sample collector was found significant factors ( $\mathrm{p}=0.043$ and 0.018$)$.

\section{Discussion}

Definitive diagnosis in COVID-19 is made by showing SARSCoV-2 viral RNA by RT-PCR test in the samples which pharyngeal or nasopharyngeal swabs, sputum, urine, stool [11]. Pharyngeal virus shedding was detected very high during the first week of the symptoms and decline towards the end of the first week [11]. The current literatüre was reported that the incubation period of the virus changes between 3 to 14 days [12,13]. Wölfel, et al. [11] detected $16.6 \%$ virus RNA in swabs in the first week of onset of symptoms in nine patients known close contact with patients diagnosed with COVID-19, In addition, in this study, the initial symptoms were flu-like symptoms in $66.6 \%(n=6)$ of patients, while they found an odor disorder in $44 \%(n=4)$ of patients. In this study, $60.2 \%(n=41)$ of the patients had at least one rhinological symptom and 24 patients had a complaint of smell loss (35.3\%).

Table 1: Frequency and percentage of symptoms.

\begin{tabular}{|c|c|c|}
\hline Symptoms & N & \% \\
\hline Cough & 48 & 70,6 \\
\hline Dyspnea & 39 & 57,4 \\
\hline Fever & 39 & 57,4 \\
\hline Fatigue & 26 & 38,2 \\
\hline Sore throat & 25 & 36,8 \\
\hline Headache & 24 & 35,3 \\
\hline Nasal Discharge & 17 & 25,0 \\
\hline Nasal Obstruction & 13 & 19,1 \\
\hline Sneeze & 6 & 8,8 \\
\hline
\end{tabular}

Table 2: Frequency and percentage of findings.

\begin{tabular}{|c|c|c|c|}
\hline Variables & & $\mathbf{N}$ & \% \\
\hline \multirow{2}{*}{ Gender } & Female & 30 & 44,1 \\
\cline { 2 - 4 } & Male & 38 & 55,9 \\
\hline
\end{tabular}




\begin{tabular}{|c|c|c|c|}
\hline \multirow{2}{*}{ ARD } & - & 53 & 77,9 \\
\hline & + & 15 & 22,1 \\
\hline \multirow{2}{*}{ NSU } & - & 56 & 82,4 \\
\hline & + & 12 & 17,6 \\
\hline \multirow{2}{*}{ LPR } & - & 53 & 77,9 \\
\hline & + & 15 & 22,1 \\
\hline \multirow{2}{*}{ Sample collectors } & OP & 47 & 69,1 \\
\hline & ORL & 21 & 30,9 \\
\hline \multirow{2}{*}{ RT-PCR } & - & 38 & 55,9 \\
\hline & + & 30 & 44,1 \\
\hline
\end{tabular}

Table 3: Distribution of variables according to RT-PCR test results and $p$-values (X2).

\begin{tabular}{|c|c|c|c|c|c|c|}
\hline & & \multicolumn{5}{|c|}{ RT-PCR } \\
\hline & & \multicolumn{2}{|c|}{+} & \multicolumn{2}{|c|}{-} & $\mathbf{P}$ \\
\hline & & $\mathbf{N}$ & $\%$ & $\mathbf{N}$ & $\%$ & \\
\hline \multirow{2}{*}{ Gender } & Female & 12 & 17,6 & 18 & 26,5 & \multirow{2}{*}{0.543} \\
\hline & Male & 18 & 26,5 & 20 & 29,4 & \\
\hline \multirow{2}{*}{ ARD } & - & 24 & 35,3 & 29 & 42,6 & \multirow{2}{*}{0.716} \\
\hline & + & 6 & 8,8 & 9 & 13,2 & \\
\hline \multirow{2}{*}{ NSU } & - & 28 & 41,2 & 28 & 41,2 & \multirow{2}{*}{0.035} \\
\hline & + & 2 & 2,9 & 10 & 14,7 & \\
\hline \multirow{2}{*}{ LPR } & - & 27 & 39,7 & 26 & 38,2 & \multirow{2}{*}{0.033} \\
\hline & + & 3 & 4,4 & 12 & 17,6 & \\
\hline \multirow{2}{*}{ Sample collectors } & OP & 16 & 23,5 & 31 & 45,6 & \multirow{2}{*}{0.012} \\
\hline & ORL & 14 & 20,6 & 7 & 10,3 & \\
\hline
\end{tabular}

Wang et al. [14] have reported that the SARS-CoV-2 RNA was detected only in $32 \%$ of pharyngeal swabs $(126 / 398)$ and in $63 \%$ of nasopharyngeal swabs (5/8). However, this report does not mention the collection time of the samples or the onset of symptoms [14]. Samples were collected in the first week ( $\mathrm{m} \pm \mathrm{SD}=3.8 \pm 2.2$ days) after the onset of symptoms and Viral RNA was detected on the 38 (55.9\%) of swab samples, also collection time was not found as a significant factor for RT-PCR results. The results are consistent with the current literature, But the timing for swab collection was not discussed clearly in referential studies.

Examples of OP/NP swabs are easy and practical methods, as well as anatomical and medical conditions that may concern this technique. Nasal steroids are frequently preferred treatment options for nasal inflammatory diseases such as allergic rhinitis $[7,15]$. The clinical effects of topical steroids increase the recruitment of inflammatory cells to the airway mucosa, selectively suppress local cytokine expression, inhibit mediator release, and support the normal mucosal structure [16]. Ogimi, et al. [17] demonstrated a significant association between prolonged shedding of human coronavirus ( $\mathrm{HCoV}$ ) with high-dose steroid use in hematopoietic cell transplant. Waltl, et al. [18] reported that betamethasone may decrease epithelial damage caused by rhinovirus infection by antiinflammatory properties on immune cells. Kim, et al. [19] showed that the antiviral activity of budesonide in vitro via inhibiting the human rhinovirus replication by autophagy activation and suggest the budesonide a therapeutic option.

Yamaya, et al. [10] demonstrated that budesonide reduces rhinovirus count, replication, and concentrations of cytokines of primary cultures of human tracheal epithelial cells. The difference of RT-PCR positiveness detection on swabs (2.9\% vs. $41.2 \%$ ) in NS cases with COVID-19 patients can be explained by antiinflammatory or antiviral effects of steroids. Although the number of studies on $\mathrm{HCoV}$ and steroid use is low and current studies are related to rhinovirus. A significantly high RT-PCR negativity in the NSU group in this study can be explained with the existing data of the literature. Although, the use of the different types of nasal steroids, dose, and the absence of histopathological evidence are limitations of this study. The significant-high difference for ORL among physicians probably was depended on the anatomical knowledge of the ORL group and was not a surprise.

LPR dependent mucosal changes have been previously described in the literature [4]. Sella, et al. [20] showed in the literature review that LPR has a pathological effect on the nasal mucosa in patients with chronic rhinosinusitis disease. However, the effects of LPR disease on respiratory tract viral infection are unclear and no study was found in a literature review. In this study, SARS-CoV-2 RT-PCR negativity was significantly increased in OP/ NP swabs in patients with LPR. However, the validity of this finding is controversial due to the small sample size and its absence of histopathological findings. 


\section{Conclusion}

During the COVID-19 pandemic, rapid and early diagnosis of the disease is essential for the isolation of patients and prevent transmission. OP/NP swaps are the most widely used samples for the diagnosis of SARS-CoV-2. With the reasonable data in the current literature and results of this study, it must be considered that chronic nasal steroid use may affect RT-PCR test results. Also, virus detection on swabs may depend on the sampling technique. Educational interviews by an ORL may be beneficial for swab collections and increase the rate of true positivity. Thus, repetitive tests can be reduced.

\section{Acknowledgement}

None.

\section{Conflict of Interest}

The authors declare that they have no conflict of interest.

\section{References}

1. Sun P, Lu X, Xu C (2020) Understanding of COVID-19 based on current evidence. J Med Virol 92(6):548-551.

2. Cao Y, Liu X, Xiong L (2020) Imaging and Clinical Features of Patients With 2019 Novel Coronavirus SARS-CoV-2: A systematic review and meta-analysis. J Med Virol 1002.

3. Loeffelholz MJ, Tang YW (2020) Laboratory Diagnosis of Emerging Human Coronavirus Infections-The State of the Art. Emerg Microbes Infect 1751: 1-26.

4. Powell J, Cocks HC (2013) Mucosal changes in laryngopharyngeal reflux-Prevalence, sensitivity, specificity and assessment. Laryngoscope 123(4): 985-991.

5. Lupa M, DelGaudio JM (2012) Evidence-Based Practice. Reflux in Sinusitis. Otolaryngol Clin North Am 45(5): 983-992.

6. Dagli E, Yüksel A, Kaya M (2017) Association of oral antireflux medication with laryngopharyngeal reflux and nasal resistance. JAMA OtolaryngolHead Neck Surg 143(5): 478-483.

7. Wise SK, Lin SY, Toskala E (2018) International consensus statement on allergy and rhinology: allergic rhinitis-executive summary. Int Forum Allergy Rhinol 8(2): 85-107.
8. Feng CH, Miller MD, Simon RA (2012) The united allergic airway: Connections between allergic rhinitis, asthma, andchronic sinusitis. Am J Rhinol Allergy 26(3): 187-190.

9. Lan F, Zhang N, Gevaert E (2016) Viruses and bacteria in Th2-biased allergic airway disease. Allergy Eur J Allergy Clin Immunol 71(10): 1381-1392.

10. Yamaya M, Nishimura H, Nadine L (2014) Formoterol and budesonide inhibit rhinovirus infection and cytokine production in primary cultures of human tracheal epithelial cells. Respir Investig 52(4): 251-260.

11. Wölfel R, Corman VM, Guggemos W (2020) Virological assessment of hospitalized patients with COVID-2019. Nature 465-469.

12. Gralinski LE, Menachery VD (2020) Return of the coronavirus: 2019nCoV. Viruses 12(2): 1-8.

13. Dhungana HN (2020) Comments on "Preliminary estimation of the basic reproduction number of novel Coronavirus (2019-nCoV) in China, from 2019 to 2020: A data-driven Analysis in the early phase of the outbreak." Int J Infect Dis 94: 72-73.

14. Wang W, Xu Y, Gao R (2020) Detection of SARS-CoV-2 in Different Types of Clinical Specimens. JAMA 323(18): 1843-1844.

15. Szefler SJ (2001) Pharmacokinetics of intranasal corticosteroids. J Allergy Clin Immunol 108(1): S26-31.

16. Minshall E, Ghaffar O, Cameron L (1998) Assessment by nasal biopsy of long-term use of mometasone furoate aqueous nasal spray (Nasonex) in the treatment of perennial rhinitis. Otolaryngol-Head Neck Surg 118(5): 648-654.

17. Ogimi C, Greninger AL, Waghmare AA (2017) Prolonged shedding of human coronavirus in hematopoietic cell transplant recipients: Risk factors and viral genome evolution. J Infect Dis 216(2): 203-209.

18. Waltl EE, Selb R, Eckl-Dorna J (2018) Betamethasone prevents human rhinovirus- and cigarette smoke- induced loss of respiratory epithelial barrier function. Sci Rep 8(1): 1-10.

19. Kim SR, Song JH, Ahn JH (2017) Antiviral and anti-inflammatory activity of budesonide against human rhinovirus infection mediated via autophagy activation. Antiviral Res 151: 87-96.

20. Sella CGP, Tamashiro E, Anselmo-lima WT (2017) Relation between chronic rhinosinusitis and gastroesophageal reflux in adults: systematic review. Braz J Otorhinolaryngol (83(3): 356-363. 